\title{
Position Based Balloon Angioplasty
}

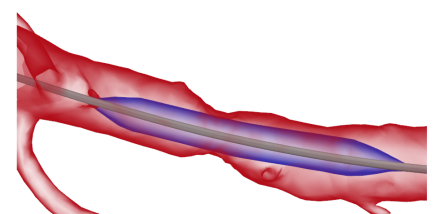

(a) $\mathrm{T}=0.000 \mathrm{~s}$

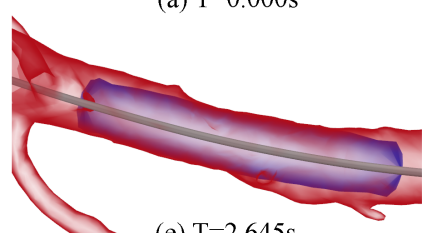

(e) $\mathrm{T}=2.645 \mathrm{~s}$

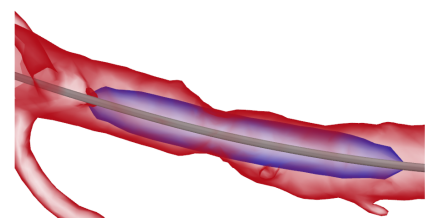

(b) $\mathrm{T}=0.555 \mathrm{~s}$

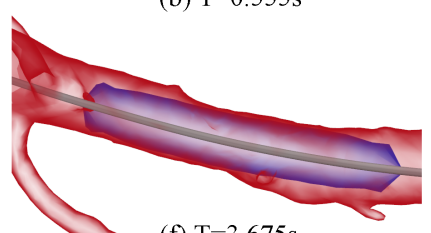

(f) $\mathrm{T}=3.675 \mathrm{~s}$

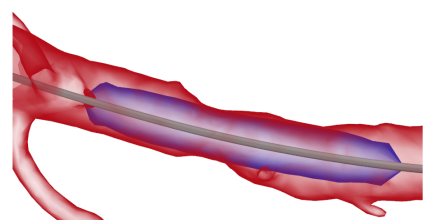

(c) $\mathrm{T}=1.150 \mathrm{~s}$

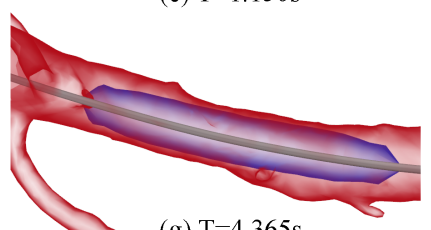

(g) $\mathrm{T}=4.365 \mathrm{~s}$

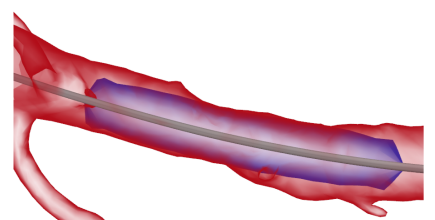

(d) $\mathrm{T}=1.855 \mathrm{~s}$

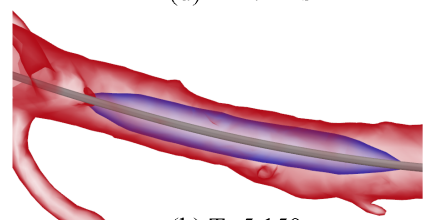

(h) $\mathrm{T}=5.150 \mathrm{~s}$

Figure 1: The real lesion coronary artery dilated by the inflation balloon

\begin{abstract}
Balloon angioplasty is an endovascular procedure to widen narrowed or obstructed blood vessels, typically to treat arterial atherosclerosis. Simulating angioplasty procedure in the complex vascular structures is a challenge task since the balloon and vessels are both flexible bodies. In this paper, we proposed a position based balloon physical model to solve nonlinear physical deformation in the process of balloon inflation. Firstly, the balloon is discrete modeled by the closed triangle mesh, and the hyperelastic membrane material and continuum based formulation are combined to compute the mechanical properties in the process of balloon inflation. Then, an adaptive air mesh generation algorithm is proposed as a preprocessing procedure for accelerating the coming collision process between balloon and blood vessel according to the characteristic of collision area which is relative fixed. The experiment results show that this physical model is feasible, which could simulate the contact and deformation process between the inflation balloon and the diseased blood vessel wall with good robustness and in realtime.
\end{abstract}

Keywords: Physical simulation, Balloon angioplasty, Continuum mechanics, Position based method

\section{Introduction}

Cardiovascular diseases are the number one cause of death in the world. Among them, Atherosclerosis is the most common disease, which is much harmful to human health. This kind of cardiovascular diseases are mainly caused by the cholesterol, cellular waste, calcium and fibrous protein which are clotting and forming plaques inside the vessel [Naghavi et al. 2003]. These plaques block the blood vessels which decreasing the blood flow and oxygen delivery, finally, results in acute death such as cardiac death or brain death etc.

Balloon angioplasty is the most common and effective minimally invasive vascular surgery to cure this arteriolar occlusion, and it is the important step before intravascular stent implantation. Firstly, the balloon-catheter reaches the lesion location in the stenosed artery along the guidewire. Then, the outer pressure injector inflates balloon, at the same time, the balloon dilates the artery which has been clogged up by atheromatous plaque, or dilates the stent to ex- pand the lumen area of stenosed artery. Through those treatments, the blood flow of dilated artery can recover to the acceptable levels.

Balloon is an important surgical instrument in the balloon angioplasty, the simulation of the inflation balloon is a challenging problem. 1) The thickness of balloon membrane changed with the inflation of the balloon, which means needing a physical model similar with real balloon to simulate the mechanical properties of balloon. 2) Balloon and blood vessels both are flexible bodies, the vascular model has a large number of meshes and complex structure, it is hard to complete the collision detection and response between them in real-time.

To deal with above problems, we firstly introduce the continuum mechanics into the physical modeling of the balloon. For the real-time performance on collision detection between two flexible soft objects, the balloon and blood vessels, we proposed a collision mesh preprocess method. Compared with the existing balloon physical model, we consider the mechanical properties of real balloon and adds the thickness property into the plane meshes. Meanwhile, the locality of collision region is used to generate the adaptive collision air meshes between balloon and blood vessels, and speed up their collision handling. The experimental results show that our balloon model can satisfy the real-time requirement of virtual balloon angioplasty, and able to apply to the virtual minimally invasive vascular surgery training system.

\section{Related Work}

Balloon angioplasty is an important part of virtual invasive surgery, but there are few studies about virtual balloon angioplasty surgery for the training system at present. Cai Y et al. [Cai et al. 2003] described a virtual surgery simulation system, and the inflation of balloon is showed in this system, but it did not involve the related technologies in detail. Later, they proposed a hand-eye coordination haptic VR system [Cai et al. 2006] to simulate the percutaneous transluminal coronary angioplasty. Luboz $\mathrm{V}$ et al.[Luboz et al. 2014] introduced a stent and balloon simulation system, in which the balloon is indicated as a series of overlapping particles attaching to the guidewire, the dilation of these particles express the dilation of balloon. This particle based method neither simulates the dynamics processes of balloon inflation and deflation accurately, nor does it simulate the multi-contact collision between balloon and blood vessel. When the balloon contacting with the blood ves- 
sel, they adopted a serial of threshold values of inflation balloon to trigger corresponding deformation state of the blood vessel as the response of contact collision. This method using multi-level of blood vessel can accelerate the collision detection, however, the generation of different levels of blood vessel are a verbose thing. Moreover, it can only simulate the deformation of blood vessel at the fixed position on the generated blood vessel. Finally, this responding way of collision ignores the process of dynamics collision interaction between balloon and blood vessel.

Physical model: Balloon is a hyperelastic membrane, there are already many medical numerical simulation analyses of balloon angioplasty [Gasser and Holzapfel 2007; Zahedmanesh et al. 2010], and finite element method(FEM) is adopted in those numerical simulation analyses to simulate and model the balloon and blood vessel. This method has high precision but low simulation speed, therefore, it is hard to apply to the real-time simulation training system. Terzopoulos et al. [Terzopoulos et al. 1987] firstly introduced the simulation of deformable body into computer graphics, and they adopted the finite difference method to simulate the deformable body and thin plate. Later, the simulation of deformable body became an active research field and many relevant simulation methods were proposed. In this paper we mainly focus on the relevant simulation methods about membrane, thin shell and balloon.

Wu et al. [Wu et al. 2001] solved the continuum mechanics function by FEM to simulate the nonlinear deformation of the membrane. Based on Kirchhoff-Love thin shell theory, Cirak et al. [Cirak et al. 2002] adopted subdivision surface to simulate the thin shell structure in FEM. Wang et al. [Wang et al. 2015] adopted three prism element to construct the surface of balloon and compute the large deformation of balloon inflation based on FEM. These FEM-based methods can accurately simulate the nonlinear deformation of the membrane, but hardly achieve real-time performance. Choi and Ko [Choi and Ko 2005] proposed thin material cloth simulation method based on mass-spring model. This mass-spring method is hard to simulate the required state of real model due to the stiffness of the spring is difficult to obtain. Moreover, Grinspun et al. [Grinspun et al. 2003] proposed the thin shell discrete model to simulate the thin flexible structures, such as hat and paper. And Tang et al. [Tang et al. 2015] extended strain-limiting method and proposed multiresolution method to real-time simulate the thin elastic materials. The discrete thin shell model determines it is hard to simulate the specified material. To realistically simulate the deformation of the real material, the continuum material model is widely adopted to simulate the behavior of the mentioned real deformable material. Müller et al. [Müller et al. 2004] adopted the point-based method and combined the continuum mechanics to simulate the elastic and plastic body. Volino et al. [Volino et al. 2009] used the continuum mechanics to simulate the cloth membrane. For fabricationoriented goals, Skouras et al. [Skouras et al. 2012] modeled the rubber material balloon by the method of continuum mechanics with constant thickness of the balloon, and they selected the material model by fitting the experiment data. Later, they have simulated the wrinkling of inflatable membrane by the tension field theory [Skouras et al. 2014]. Bender et al. [Bender et al. 2014] introduced the position-based method and combined the continuum mechanics to real-time robustly simulate the deformable body and plane cloth without thickness. Recently, Bouaziz et al. [Bouaziz et al. 2014] proposed projective dynamics method, which is similar with the position-based method, to simulate the solid, cloth, membrane, etc.

Collision detection: Up to now, a great number of Collision detection algorithms has been proposed. The computation of collision detection becomes expensive with plenty of the space-mesh, and many acceleration algorithms have been put forward to improve the efficiency of collision detection. The spatial hashing method proposed by Teschner et al. [Teschner et al. 2003], Tang et al. [Tang et al. 2011] presented a separating axis tests method, Barbič and James [Barbič and James 2010] and Zheng and James [Zheng and James 2012] came up with a simulation space based culling method, and Mezger et al. [Mezger et al. 2003], Schvartzman et al. [Schvartzman et al. 2010] and Schvartzman et al. [Schvartzman et al. 2009] put forward multi-hierarchical methods.

For the collision detection of deformable body, omitting single collision always lead to entangled state between two objects which is difficult to separate later. Bridson et al. [Bridson et al. 2002] proposed a robust collision handling algorithm to deal with the collision of cloth by introducing the continuous collision detection(CCD). Thereafter, many CCD optimization methods [Zhang and Kim 2012; Wang 2014; Tang et al. 2014] have been came up. Sometimes, the deformable bodies collide and contact with each other forcedly which causes these objects into entangled state, such as the the collision and extrusion between the balloon and blood vessels. However, the CCD method cannot solve this problem well. To address this problem, Baraff et al. [Baraff et al. 2003] used the separate force to untangle the intersected meshes by analysing the structure of intersection meshes, and Wicke et al. [Wicke et al. 2006] extended this method to handle the boundary of mesh. Volino et al. [Volino and Magnenat-Thalmann 2006] introduced a contour minimization method to untangle the intersected surfaces. Another solution to the untangling problem is to handle the meshes before intersection. Such a history-based method was proposed by Selle et al. [Selle et al. 2009] to solve the force apply on the collision pairs, but this method need to reinitialize the memory of mesh state for every time step. Müller et al. [Müller et al. 2015] handled this problem by generating the space collision tetrahedron, and this method can robustly simulate the collision of large mesh in realtime with initializing once before the simulation.

Since Müller et al. [Müller et al. 2007] proposed the position based dynamics to simulate the deformable objects, such as cloth and balloon etc., recent years the position based method [Bender et al. 2014; Macklin et al. 2014; Müller et al. 2015] became a topic of active research. This kind of solving method has been widely used due to its efficiency and stability in the physical simulation of various objects. Therefore, based on the previous work [Tang et al. 2010] we introduce the position based method to simulate the entire process of the balloon angioplasty. For the modeling of the balloon, we adopt the position based method and combine the continuum mechanics to fast and stably simulate the nonlinear deformation of the balloon in the blood vessels. Before inflation of the balloon, we introduce an adaptive air mesh generation algorithm to fast generate the inner tetrahedron mesh between the balloon and blood vessels. Finally, we handle the collision between the balloon and complex blood vessels based on the air mesh method [Müller et al. 2015].

\section{Nonlinear continuum material physical model}

Nonlinear continuum mechanics is widely adopted to accurately simulate the deformation of various materials. The physical model of balloon is the key in the balloon angioplasty, and the angioplasty result will be influenced by the inflated balloon material. The balloon is a kind of rubber-like membrane with the changing thickness under inflation. Therefore, we introduce the continuum mechanical approach to real-time simulate the nonlinear physical deformation of the inflation balloon. 


\subsection{Mechanics}

The balloon will be inflated several times by the injected air or fluid under pressure, and it also can return to its original state after deflation. Following we will describe the mechanical property of this large deformation. The $\overline{\mathbf{x}}$ donates the position of the particle at initial undeformation state, $\mathbf{x}$ donates the position of the particle ar deformation state. And their mapping relation of these two states is:

$$
\mathbf{x}=\phi(\overline{\mathbf{x}}, t))
$$

From this mapping, the corresponding deformation gradient is:

$$
\mathbf{F}=\frac{\partial \mathbf{x}}{\partial \overline{\mathbf{x}}}
$$

Then the $2 \times 2$ right Cauchy-Green tensor can be defined as:

$$
\mathbf{C}=\mathbf{F}^{T} \mathbf{F}
$$

Therefore, the first and third invariants of the tensor are:

$$
\begin{gathered}
I_{1}=\operatorname{tr}(\mathbf{C}) \\
I_{3}=\operatorname{det}(\mathbf{C})
\end{gathered}
$$

We assume the simulation material does not exist transverse shear strain and satisfy incompressible assumption, then the thickness of current state is:

$$
h=H \sqrt{I_{3}^{-1}}
$$

where the $H$ is the thickness of initial undeformed state, the physical significance of $I_{3}$ is the fraction of volume change.

Therefore, the deformation energy can be integrated by the strain energy density function $\Psi(\mathbf{C})$ in the deformation domain:

$$
E=\int_{\Omega} \Psi(\mathbf{C}) d \mathbf{x}
$$

Since the energy density function determines the physical property of material, we need to determine the simulation material of the balloon and corresponding material's coefficient to realistically simulate the physical deformation of the balloon.

\subsection{Hyperelastic Balloon membrane material}

The medical balloon almost made by the polyurethane material, and there are already many numerical simulation methods adopted the Mooney-Rivlin material model to simulate the polyurethane rubber type material [Chua et al. 2003; Eftaxiopoulos and Atkinson 2005; Karimi et al. 2014]. Chua et al. [Chua et al. 2003] adopted the nonlinear analysis method to determine the Mooney-Rivlin material model to represent the material of balloon and its coefficients. Therefore, we adopt the Mooney-Rivlin material model to simulate our balloon, and its strain energy density function is:

$$
\begin{gathered}
\Psi=C_{1}\left(\bar{I}_{1}-3\right)+C_{2}\left(\bar{I}_{2}-3\right) \\
\bar{I}_{1}=\lambda_{1}^{2}+\lambda_{2}^{2}+\lambda_{3}^{2} \\
\bar{I}_{2}=\lambda_{1}^{-2}+\lambda_{2}^{-2}+\lambda_{3}^{-2}
\end{gathered}
$$

where $\bar{I}_{1}$ and $\bar{I}_{2}$ are first and second stretch invariants, the coefficients $C_{1}$ and $C_{2}$ are derived using test data which represents all modes of deformation and strain ranges. The Table 1 shows the coefficients of this material.

Table 1: Parameters of the Mooney-Rivlin Material

\begin{tabular}{c|c|c|c}
\hline Material & Density $\left(\mathrm{kg} / \mathrm{mm}^{3}\right)$ & $C_{1}$ & $C_{2}$ \\
\hline Polyurethane & $1.07 \mathrm{E}-6$ & 1.031 & 3.69 \\
\hline
\end{tabular}

The $\lambda_{1}$ and $\lambda_{2}$ in Equation(9) and (10) are the squares of the principal 'in-plane' stretches, and the values of stretch are the eigenvalue of the right Cauchy-Green tensor $\mathbf{C}$ :

$$
\begin{aligned}
& \lambda_{1}^{2}=\frac{1}{2}\left(I_{1}+\left(I_{1}^{2}-4 I_{3}\right)^{1 / 2}\right) \\
& \lambda_{2}^{2}=\frac{1}{2}\left(I_{1}-\left(I_{1}^{2}-4 I_{3}\right)^{1 / 2}\right)
\end{aligned}
$$

$\lambda_{3}$ is the thickness stretch which can be obtained by the incompressible condition:

$$
\begin{gathered}
\lambda_{1} \lambda_{2} \lambda_{3}=1 \\
\lambda_{3}=\left(\lambda_{1} \lambda_{2}\right)^{-1}=I_{3}^{-1 / 2}
\end{gathered}
$$

\subsection{Discretization}

We adopt the closed triangle mesh and linear Lagrangian shape function to discrete the balloon model. For a closed balloon triangle mesh model, $\overline{\mathbf{x}}_{i}$ donates the position of i-th vertex at undeformed state, $\mathbf{x}_{i}$ donates the position of i-th vertex at the deformed state. And the corresponding edge vector of undeformed state and deformed state are $\overline{\mathbf{e}}_{i j}=\overline{\mathbf{x}}_{j}-\overline{\mathbf{x}}_{i}$ and $\mathbf{e}_{i j}=\mathbf{x}_{j}-\mathbf{x}_{i}$ respectively. For a initial state of the triangle, we firstly construct the material frame matrix of this triangle as $\overline{\mathbf{R}}=[\overline{\mathbf{u}} \mathbf{\mathbf { v }}]$, where $\overline{\mathbf{u}}=\frac{\left(\overline{\mathbf{e}}_{12} \times \overline{\mathbf{e}}_{13}\right) \times \overline{\mathbf{e}}_{12}}{\left|\left(\overline{\mathbf{e}}_{12} \times \overline{\mathbf{e}}_{13}\right) \times \overline{\mathbf{e}}_{12}\right|}$ and $\overline{\mathbf{v}}=\frac{\overline{\mathbf{e}}_{12}}{\left|\overline{\mathbf{e}}_{12}\right|}$.

The deformation gradient of a deformed triangle is $\mathbf{F}=\mathbf{D}_{s} \mathbf{D}_{m}^{-1} \in$ $\mathbb{R}^{3 \times 2}$, where $\mathbf{D}_{s}=\left[\begin{array}{ll}\mathbf{e}_{31} & \mathbf{e}_{32}\end{array}\right]$ is the deformed shape matrix, and $\mathbf{D}_{m}=\left[\begin{array}{l}\overline{\mathbf{e}}_{31} \\ \overline{\mathbf{e}}_{32}\end{array}\right] \overline{\mathbf{R}}$ is the constant reference shape matrix. Therefore, we can solve the strain energy of triangle element $E(\mathbf{x})$ from the deformation gradient by the previous equations.

$$
E(\mathbf{x})=\int_{\Omega} \Psi(\mathbf{C}) d \mathbf{x}=\Psi(\mathbf{C}) \cdot h A
$$

where $h$ is the thickness of triangle element at current state, and $A$ is its current area. We adopt the position based energy reduction method [Bender et al. 2014] to solve this physical model, and the energy constraint function is $C(\mathbf{x})=E(\mathbf{x})=0$. In the position based method, the position of the particles are firstly estimated by a time integration step, and then the constraint functions are adopted to correct the position of particles to meet the constraint functions. The energy gradient is used to determine the correction displacement of the triangle element vertices. Then the energy gradient of Equation(15) is:

$$
\nabla_{\mathbf{x}} E=\int_{\Omega} \frac{\partial \Psi}{\partial \mathbf{x}} d \mathbf{x}=\int_{\Omega} \frac{\partial \Psi}{\partial \mathbf{F}} \frac{\partial \mathbf{F}}{\partial \mathbf{x}} d \mathbf{x}=\int_{\Omega} \mathbf{P}(\mathbf{F}) \frac{\partial \mathbf{F}}{\partial \mathbf{x}} d \mathbf{x}
$$

where the first Piola-Kirchhoff stress tensor $\mathbf{P}(\mathbf{F})$ is:

$$
\mathbf{P}(\mathbf{F})=\frac{\partial \Psi}{\partial I_{1}} \frac{\partial I_{1}}{\partial \mathbf{F}}+\frac{\partial \Psi}{\partial I_{3}} \frac{\partial I_{3}}{\partial \mathbf{F}}
$$


The every part of $\mathbf{P}(\mathbf{F})$ is derived in the Appendix. Therefore, The three strain energy gradients of the vertices of the triangle element are:

$$
\begin{gathered}
{\left[\begin{array}{cc}
\frac{\partial E}{\partial \mathbf{x}_{1}} & \frac{\partial E}{\partial \mathbf{x}_{1}}
\end{array}\right]=\mathbf{P}(\mathbf{F}) \mathbf{D}_{m}^{-T} \cdot h A} \\
\frac{\partial E}{\partial \mathbf{x}_{3}}=-\sum_{i=1}^{2} \frac{\partial E}{\partial \mathbf{x}_{i}}
\end{gathered}
$$

\section{Fast collision handling}

An adaptive air mesh generation algorithm is proposed to generate the tetrahedron air meshes between balloon and blood vessel according to the characteristic of the collision region where balloon colliding with blood vessel is relatively fixed, and we real-time simulate the collision interaction between the inflation balloon and complex vascular system based on the air mesh collision detection algorithm.

\subsection{Adaptive air mesh generation}

During the surgery simulation, when the balloon on the catheter reaches the lesion area and inflates, its position always keeps still. Therefore, we generate the tetrahedron air meshes adaptively to accelerate the collision detection between them, and the adaptive air mesh generation algorithm includes following three steps.

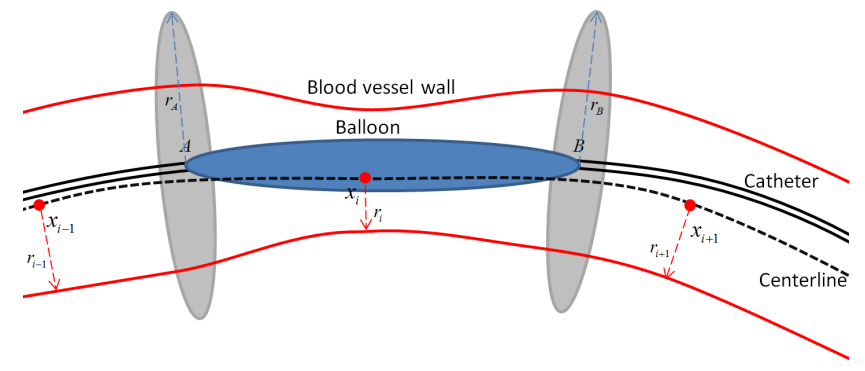

(a) Straight position

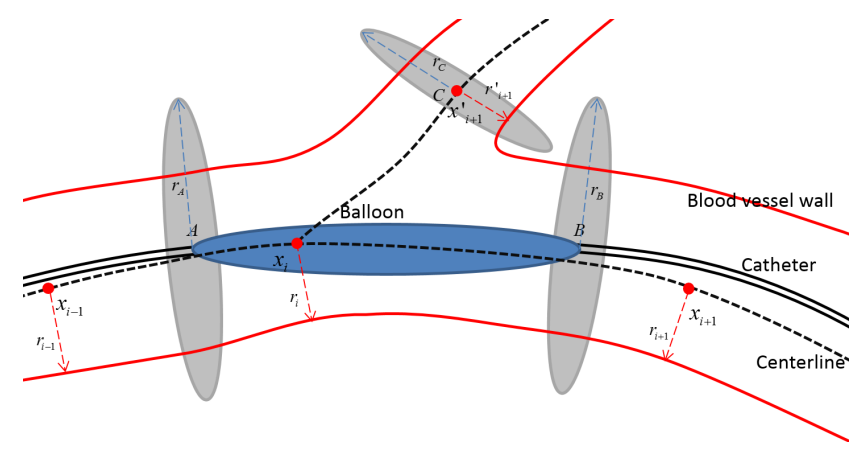

(b) Bifurcation position

Figure 2: Determine the collision area

STEP 1: According to the tubular structure of blood vessel, we use the method of interception to obtain the possible collision region between the balloon and blood vessel. (1) When the balloon is at the straight tube as shown in Fig.2(a). We respectively search for the two nearest centerline control points from the two-end of the balloon $A$ and $B$, and use the sum of radius of each adjacent centerline control point as the radius of the circular section. The nearest two centerline control points from $A$ are $x_{i-1}$ and $x_{i}$, of which the corresponding radii are $r_{i-1}$ and $r_{i}$. Therefore, the circular section radius of endpoint $A$ is $r_{A}=r_{i-1}+r_{i}$, as for $B$ is the same that $r_{B}=r_{i}+r_{i+1}$. Then the two circular sections at $A$ and $B$ could be confirmed by the tangential direction of the catheter at $A$ and $B$, which means the possible collision region is determined between them. (2) When the balloon is at the bifurcation of blood vessel, as shown in Fig.2(b). It is the same as (1) that confirming the two circular sections at $A$ and $B$ firstly. Then setting $x_{i+1}^{\prime}$ as the center point $C$ of the circular section at this bifurcation, this centerline control point is at the bifurcation of the other child, and the radius of this circular section is $r_{C}=2 r_{i+1}^{\prime}$.

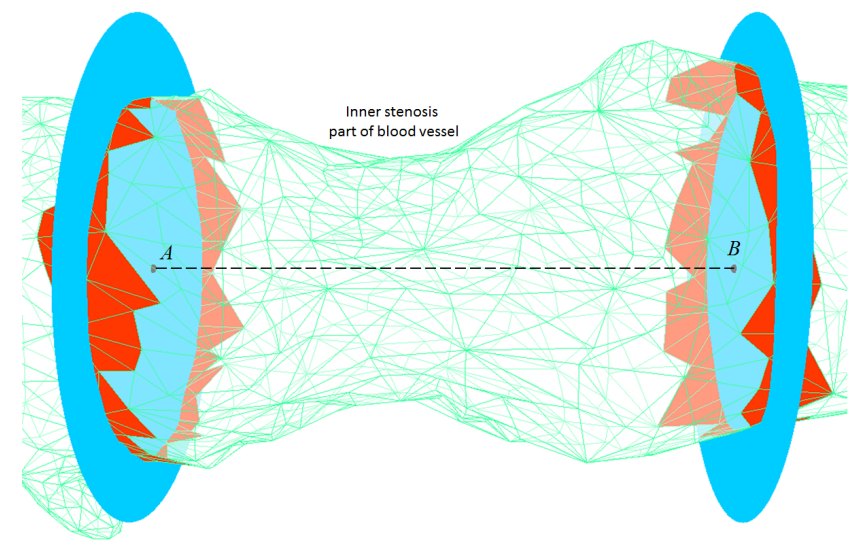

Figure 3: The inner triangle mesh of blood vessel

STEP 2: Obtaining the collision meshes of blood vessel between the circular sections, as shown in Fig.3. For convenience, we use the straight position as example, the method used at bifurcation position is the same. Firstly, we respectively compute two triangle circles (red meshes) formed by triangle patches intersecting with the circular section(blue annulus). Then, we compute the inside vertices of triangles by the signed distance to each circular section. Finally, we set the points inside the sections as the initial points, and use the propagation method iteratively get the whole inner meshes by the connectivity between mesh points.

STEP 3: Generating the air collision meshes between the blood vessel and balloon meshes. We adopt the existing tetrahedral mesh generation tool TetGen by $\mathrm{Si}$ [Si 2015] to generate the collision meshes between them.

\subsection{Air mesh collision detection}

After generated the adaptive tetrahedral collision meshes, we adop$\mathrm{t}$ unilateral constraints as well as the optimization of tetrahedral meshes to complete the collision process between balloon and vessel. The unilateral constraints equation is:

$$
C(\mathbf{x})_{a i r}=\operatorname{det}\left[\mathbf{P}_{2}-\mathbf{P}_{1}, \mathbf{P}_{3}-\mathbf{P}_{1}, \mathbf{P}_{4}-\mathbf{P}_{1}\right] \geq 0
$$

Where $\mathbf{P}_{1}, \mathbf{P}_{2}, \mathbf{P}_{3}$ and $\mathbf{P}_{4}$ are the four vertices of tetrahedral respectively. With the balloon and vessel getting closer, the volume of the corresponding tetrahedral becomes smaller. When the volume is minus $\left(C(\mathbf{x})_{\text {air }}<0\right)$, the balloon is considered to collide with blood vessel, and at this point, a positional calibration needed to be done to make the constraints equation $C(\mathbf{x})_{\text {air }} \geq 0$.

When the objects undergo large relative rotations or translation$\mathrm{s}$, the volume of tetrahedron may become 0 even if there are no collisions between objects, which causes that the objects are unable to rotate or translate normally. Thus the mesh optimization must be done to optimize the structure and quality of these tetrahedron meshes. Adopting the edge-removal and multi-face removal 
operations [Shewchuk 2002] to optimize tetrahedron optimization method, and the quality measure of tetrahedron is:

$$
\begin{gathered}
q_{\text {tetrahedron }}=\frac{12}{\sqrt{2}} \frac{V}{l_{r m s}^{3}} \\
l_{r m s}=\sqrt{\frac{l_{1}^{2}+l_{2}^{2}+l_{3}^{2}+l_{4}^{2}+l_{5}^{2}+l_{6}^{2}}{6}}
\end{gathered}
$$

Where $l_{r m s}$ is the root mean square of the lengths of six edges of the tetrahedron and $V$ is the volume of the tetrahedron.

\subsection{Contact handling}

Through the collision detection of air mesh, when the balloon collides with blood vessel, a corresponding friction force is going to be applied on each of them, and their contaction also brings viscous force.

Frictional model We set the sign of the air mesh volume as the trigger whether applying the friction or not, and for one air mesh its four vertices consist of one balloon vertex and a triangle formed by three blood vessel vertices. When the volume of air mesh meets $V_{\text {air }} \leq 0$, which means collision occurs, we calculate the tangential displacement of the balloon vertex relative to the triangle at current time step:

$$
\Delta \mathbf{x}_{\perp}=\left[\mathbf{x}_{i}^{*}-\mathbf{x}_{i}\right] \perp \mathbf{n}
$$

where $\mathbf{x}_{i}^{*}$ the new estimated position of vertex $i$ at current time step, $\mathbf{x}_{i}$ the revised position at previous time step, $\mathbf{n}$ the normal vector of the triangle. According to [Macklin et al. 2014] the displacemen$\mathrm{t}$ correction of static and dynamic friction caused by the friction being applied on the balloon vertex is:

$$
\Delta \mathbf{x}_{b a}=\frac{\omega_{b a}}{\omega_{b l}}\left\{\begin{array}{c}
\Delta \mathbf{x}_{\perp},\left|\Delta \mathbf{x}_{\perp}\right|<\mu_{s} d \\
\Delta \mathbf{x}_{\perp} \cdot \min \left(\frac{\mu_{k} d}{\left|\Delta \mathbf{x}_{\perp}\right|}, 1\right), \text { otherwise }
\end{array}\right.
$$

where $\omega_{b a}=\frac{1}{m_{b a}}$ the reciprocal quality of a balloon vertex on tetrahedron, $\omega_{b l}=\frac{1}{m_{b l 1}}+\frac{1}{m_{b l 2}}+\frac{1}{m_{b l 3}}$ the reciprocals sum of the surplus blood vessel vertices, $\mu_{s}$ the static frictional coefficient, $\mu_{k}$ the kinetic frictional coefficient and $d$ the distance threshold.

It is the same when friction being applied on the balloon, the displacement correction of three vessel vertices respectively is:

$$
\begin{aligned}
\Delta \mathbf{x}_{b l 1} & =-\frac{\omega_{b l 1}}{\omega_{b a}+\omega_{b l}} \Delta \mathbf{x}_{b a} \\
\Delta \mathbf{x}_{b l 2} & =-\frac{\omega_{b l 2}}{\omega_{b a}+\omega_{b l}} \Delta \mathbf{x}_{b a} \\
\Delta \mathbf{x}_{b l 3} & =-\frac{\omega_{b l 3}}{\omega_{b a}+\omega_{b l}} \Delta \mathbf{x}_{b a}
\end{aligned}
$$

Viscous model The surfaces of balloon and blood vessel both are wet surfaces. When they contact and collide with each other, there will produce non-negligible viscous forces. During the collision detection, the optimization of tetrahedron makes the air meshes between the balloon and blood vessel keep a good structure all the time, that is, the vertices on the balloon are front to the triangle face of the blood vessel. Therefore, the viscous item is triggered, but does not apply, when the balloon vertex moves to evoke the volume of air mesh to meet $V_{a i r} \leq V_{t h 1}$, and the air mesh is constructed the same as described in frictional model. Once the viscous item is triggered and the current volume of air mesh satisfies $V_{t h 1}<V_{a i r}<V_{t h 2}$, we add the volume constraint $C_{a i r}=V_{a i r} \leq V_{t h 1}$ to the tetrahedron to express its viscous force, and in this constraint we use the positive and negative normal direction of triangle to represent the viscous force direction of each part respectively. Finally, we cancel the viscous item constraint when the volume of the air mesh meets $V_{a i r} \geq V_{t h 2}$.

\section{Implementation}

\subsection{Inflation of the balloon}

The balloon is generally inflated by the injection air or fluid. The simulation of the fluid and its interaction between the balloon are pretty complex which is hard to simulate in real-time. Therefore, we adopt the simpler method to inflate the balloon by the inner air tetrahedron of the balloon, as shown in Fig.4, the orange nodes are the guidewire nodes, and a guidewire node and a balloon triangle construct an air tetrahedron. We compute all balloon triangle meshes to the nearest guidewire node to generate all air tetrahedrons.

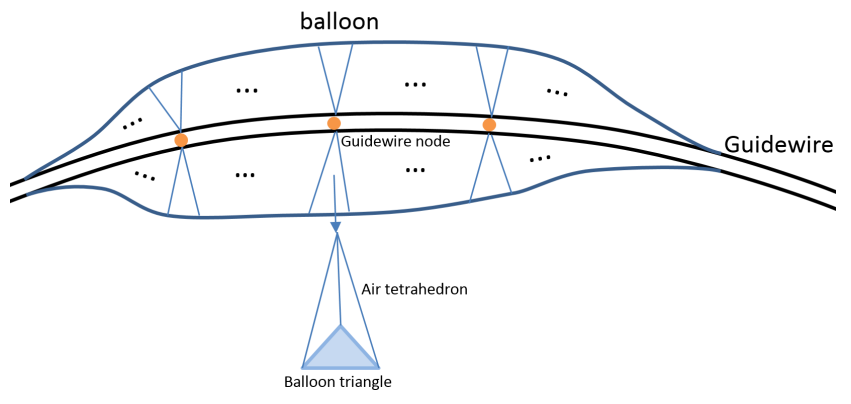

Figure 4: The inner triangle mesh of blood vessel

When inflating the balloon, the air tetrahedrons are inflating to dilate the balloon. According to the gas flow, $P V=n R T$, in which the $P$ is the pressure, $V$ is the volume, $n$ is the amount of air, $R$ is a constant, and $T$ is the temperature. Where we set the $T$ as a constant in the blood vessel. Therefore, the pressure $P_{i}$ in i-th air tetrahedron is expressed as $P_{i}=\frac{k Q_{i}}{V_{i}}$, where the $\mathrm{k}$ is a constant coefficient, $Q_{i}$ is the air mass of the i-th air tetrahedron, and $V_{i}$ is its volume. When the balloon needs to inflation, the air mass of every air tetrahedrons is increasing with a constant speed. Then the current pressure is different from the outside pressure $P_{\text {blood }}$, and we set the average blood pressure $10 \mathrm{kPa}$ in the coronary artery. Finally, we solve the pressure constraint as $C_{\text {air_tet }}=P_{i}-P_{\text {blood }}=0$ by the position-based method.

After setting the air tetrahedron, since its volume is always larger than 0 , then the interaction between the balloon and the guidewire can be easily solved as the same time.

\subsection{Elastic-plastic deformation of blood vessel}

The blood vessel is a kind of elastic body and will become plasticity when large deformation occur. To fast simulate this elastic-plastic body, the shape matching method [Müller et al. 2005] is applied to the blood vessel, and the deformation region of blood vessel is inner collision triangle mesh obtained by the Section4.1, where the boundary particles are unmovable in triangle mesh.

\section{Experiment result and discussion}

\subsection{The thickness variation of the model}

To verify the thickness of our physical model, we stretched a quadratic piece of cloth of size $6 \mathrm{~m} \times 6 \mathrm{~m}$ with thickness $1 \mathrm{~mm}$, as shown in Fig.5. The rendering results are shown in top row, and the bottoms are the corresponding thickness distribution of the cloth. The initial state of the cloth is shown in Fig.5(a). And the final transverse tensile distance of the cloth, Fig.5(d), approximately increased by $35 \%$ compared with the initial state. In this period, the 

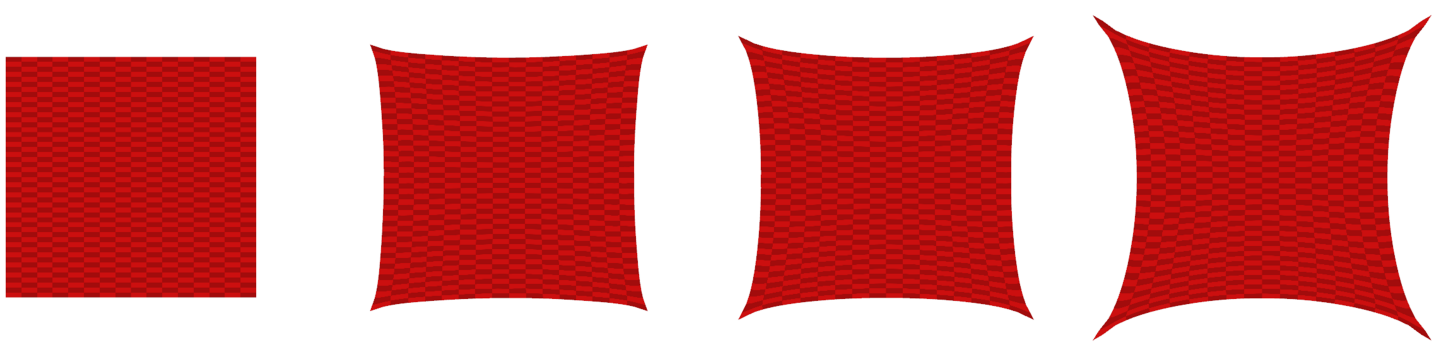

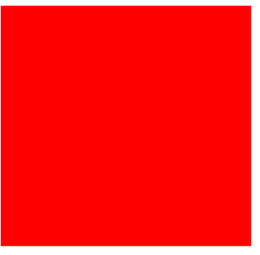

(a)

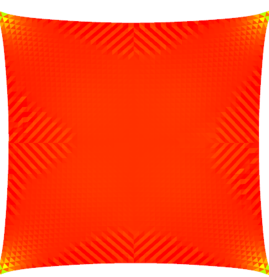

(b)

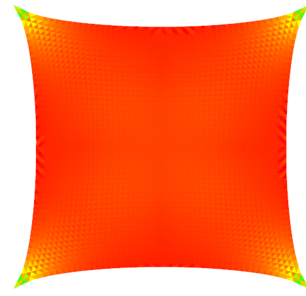

(c)

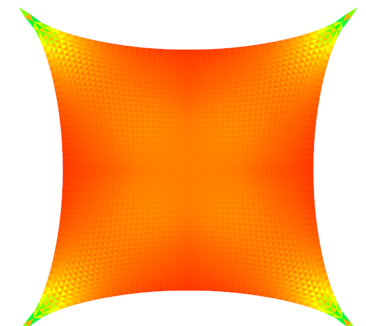

(d)

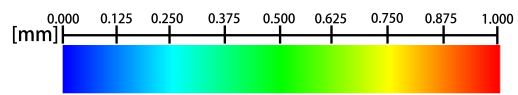

Figure 5: The quadratic piece of cloth under stretching

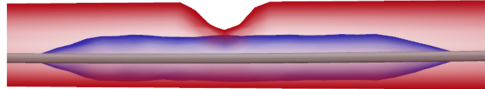

(a) $\mathrm{T}=0.000 \mathrm{~s}$

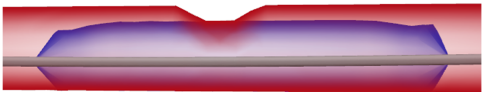

(d) $\mathrm{T}=2.190 \mathrm{~s}$

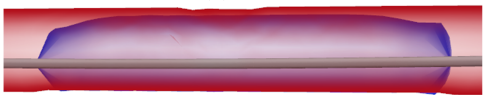

(g) $\mathrm{T}=5.830 \mathrm{~s}$

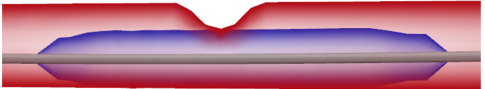

(b) $\mathrm{T}=0.640 \mathrm{~s}$

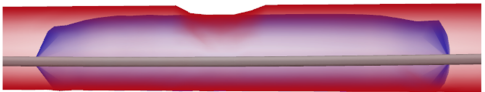

(e) $\mathrm{T}=3.170 \mathrm{~s}$

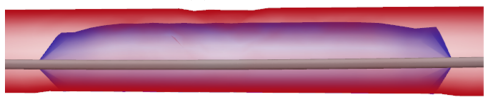

(h) $\mathrm{T}=6.940 \mathrm{~s}$

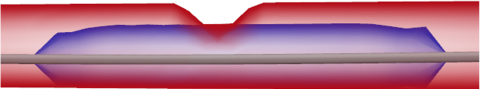

(c) $\mathrm{T}=1.360 \mathrm{~s}$

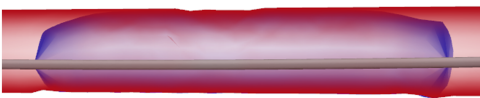

(f) $\mathrm{T}=4.505 \mathrm{~s}$

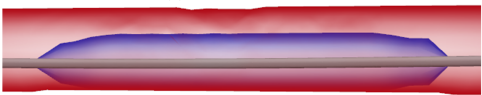

(i) $\mathrm{T}=8.395 \mathrm{~s}$

Figure 6: The regular unilateral stenosed blood vessel dilated by the inflation balloon

cloth is continuously stretched from its four corners with the same velocity, and the overall thickness of the cloth becomes thinner as shown in Fig.5(b-d). Since tensile force is applied at four corners of the cloth, the thickness around these four corners and diagonal of the cloth are thinner compared with other places.

\subsection{The inflation balloon in different type of blood ves- sel}

Human blood vessels have complex tree structure with many smaller branches. Especially, the vascular stenosis and occlusion exist in the lesion blood vessels, that makes the inner vascular structure more complicated. To verify the simulation of the balloon and blood vessels deformation process, we designed four experiments in different type of blood vessel. In the top three experiments, the regular unilateral, bilateral and Y-type of stenosed blood vessels are used to simulate the complex structure of the blood vessels. And in the fourth experiment, the patient's coronary artery is used to realistically simulate the real balloon angioplasty.

- Exp.1 The regular unilateral stenosed blood vessel.

Fig.6 shows the inflation balloon dilates the blood vessel and its

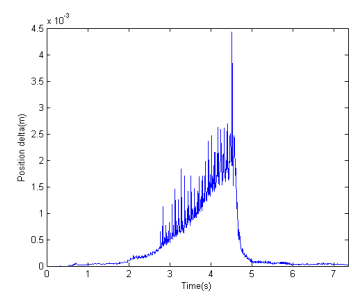

(a) frictional position delta

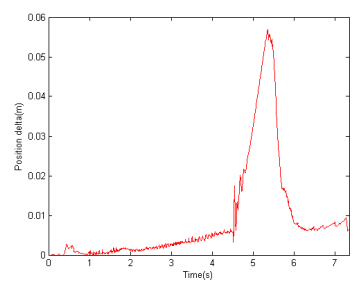

(b) viscous position delta
Figure 7: The frictional and viscous position delta of balloon in regular unilateral blood vessel

deflation, and Fig.7 shows the corresponding entire frictional and viscous position correction.

The initialized balloon is inserted into the unilateral stenosis blood vessel and reaches the stenosed region under the guidance of guidewire as shown in Fig.6(a), at this time, the frictional and viscous position delta are zero. Then, the balloon is set to inflation s- 


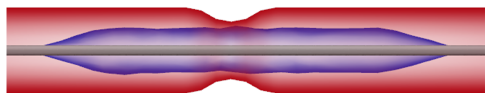

(a) $\mathrm{T}=0.000 \mathrm{~s}$

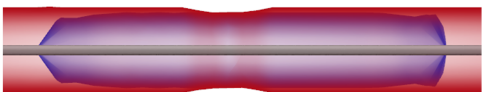

(d) $\mathrm{T}=2.580 \mathrm{~s}$

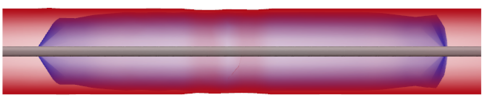

(g) $\mathrm{T}=5.000 \mathrm{~s}$

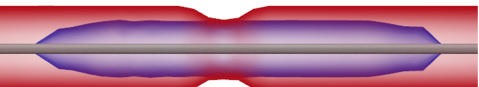

(b) $\mathrm{T}=0.575 \mathrm{~s}$

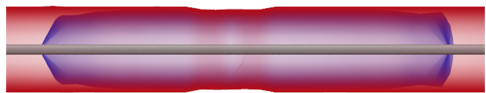

(e) $\mathrm{T}=3.285 \mathrm{~s}$

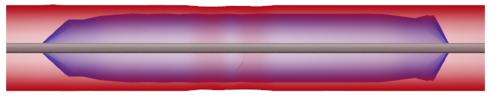

(h) $\mathrm{T}=6.190 \mathrm{~s}$

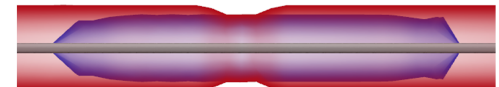

(c) $\mathrm{T}=1.470 \mathrm{~s}$

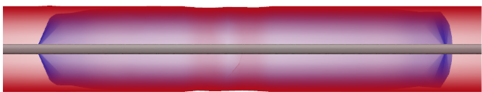

(f) $\mathrm{T}=3.915 \mathrm{~s}$

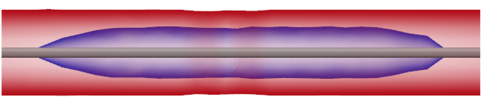

(i) $\mathrm{T}=7.605 \mathrm{~s}$

Figure 8: The regular bilateral stenosed blood vessel dilated by the inflation balloon

tate to dilate the blood vessel, and in the following time the stenosed region is extruded to the normal position, as show in Fig.6(b-f). In the this period, the frictional and viscous position delta increase gradually with the increasing contact area between the inflation balloon and the blood vessel. After the blood vessel being fully extended, the balloon is deflating for its retrieve at the time of $4.505 \mathrm{~s}$. Since then, the balloon is deflated to its original state as shown in Fig.6(g-i). During this time, the frictional position delta decreases suddenly due to the separation between the balloon and the blood vessel. Since the viscous model works when the volume of air mesh is between $V_{t h 1}$ and $V_{t h 2}$, the viscous position delta becomes larger at first and then decreases. Finally, The viscous position correction down to a certain value which is caused by the cohesion between the bottom of the balloon and blood vessel.

- Exp.2 The regular bilateral stenosed blood vessel.

The whole processes of dilating the bilateral stenosed blood vessel by the inflation balloon are shown in Fig.8, and Fig.9 shows the corresponding frictional and viscous position delta.

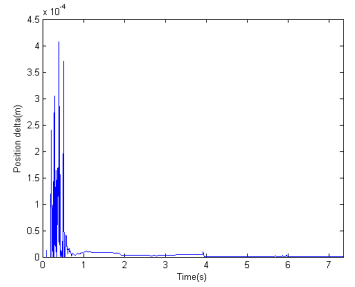

(a) frictional position delta

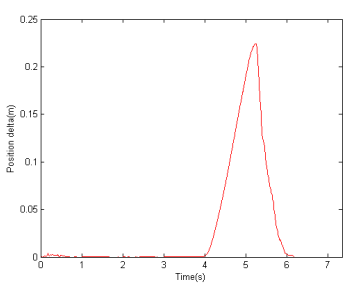

(b) viscous position delta
Figure 9: The frictional and viscous position delta of balloon in regular bilateral blood vessel

The balloon is inserted and preparing for deploying at the bilateral stenosed blood vessel at the beginning, as shown in Fig.8(a). At this time the balloon is not contacting with the blood vessel so that the frictional and viscous position delta are approximate to zero. Later, the balloon continuously inflates and contacts with the stenosed region, as shown in Fig.8(b-f). At the beginning of this time, the frictional position delta is relatively remarkable because the surface of the balloon and blood vessel start to establish contact and produce relative movement. When the blood vessel has been fully dilated, the balloon is deflating for its retrieve at the time of 3.915s. Then, the surface of balloon and blood vessel separate from each other, as shown in Fig.8(g-i). During this period, the frictional position delta is zero. And the viscous position delta becomes larger at first and then decreases down to zero which is similar with the Exp.1.

- Exp.3 The Y-type stenosed blood vessel.
The virtual balloon angioplasty treatment for the Y-type stenosed blood vessel is shown in Fig.10, and the corresponding frictional and viscous position delta are shown in Fig.11.

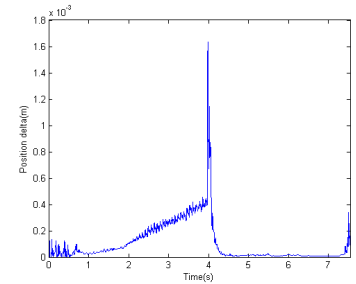

(a) frictional position delta

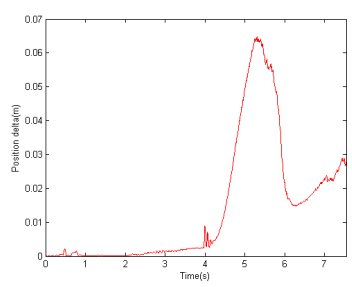

(b) viscous position delta
Figure 11: The frictional and viscous position delta of balloon in regular Y-type blood vessel

The balloon-catheter is inserted into the blood vessel and reaches the stenosis of Y-type blood vessel. Fig.10(a) shows the ballooncatheter has reached the stenosed position at the bifurcation. And then the balloon is inflating to dilate the stenosed position of the blood vessel, as shown in Fig.10(b-f). During this period, the frictional position delta increases and reaches the peak when the balloon fully contacts with the blood vessel, and then it decreases suddenly due to there are few relative movement between the balloon and blood vessel. At the time of $3.965 \mathrm{~s}$, the balloon is set to deflate for retrieve. Later, the balloon is returned to its start state as shown in Fig.10(g-i). In this time, the frictional position delta is almost zero since the detachment of the balloon and blood vessel. The viscous position delta increases firstly, then decreases, and finally it increases due to the right-upper side of balloon still clings with the blood vessel.

- Exp.4 The real lesion coronary artery.

In this experiment, the real lesion coronary artery is generated from $3 \mathrm{D}$ rotational X-ray images in DICOM dataset captured from a real patient. The balloon angioplasty results are shown in Fig.1, and the corresponding frictional and viscous position delta are shown in Fig. 11.

The balloon-catheter wrapped on the guidewire and inserted into the lesion position of the blood vessel as shown in Fig.1(a). Then, the balloon is set to the inflation state to treat the stenosed blood vessel in Fig.1(b-e). During the inflation of the balloon, the frictional position delta increases gradually and reaches the peak when the balloon is fully deployed at the time of $2.645 \mathrm{~s}$, corresponding to the Fig.1(e). At the same time, the balloon begins to deflate to its initial state, as shown in Fig.1(f-h), which leads the frictional position delta down to zero suddenly. With the balloon deflating, the 


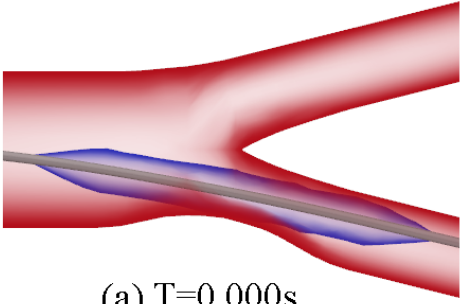

(a) $\mathrm{T}=0.000 \mathrm{~s}$

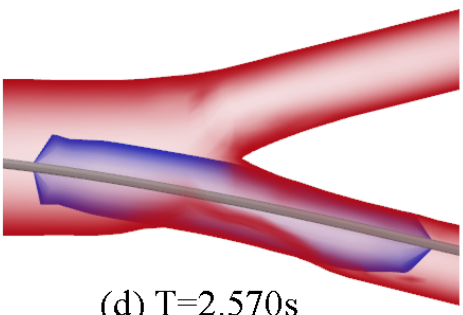

(d) $\mathrm{T}=2.570 \mathrm{~s}$

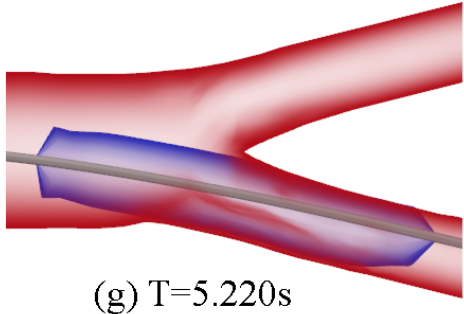

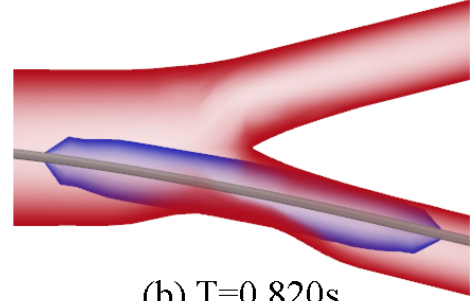

(b) $\mathrm{T}=0.820 \mathrm{~s}$

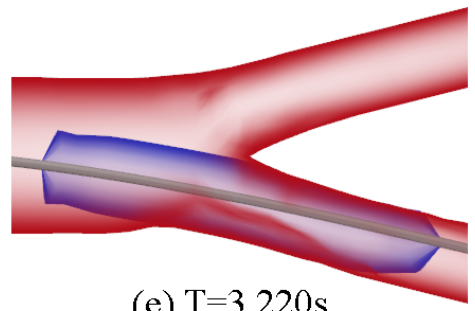

(e) $\mathrm{T}=3.220 \mathrm{~s}$

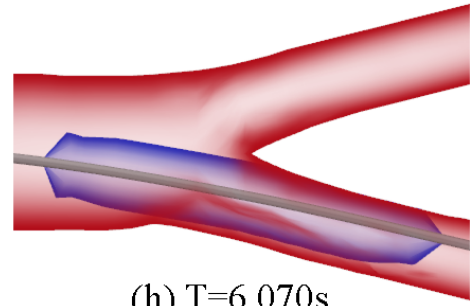

(h) $\mathrm{T}=6.070 \mathrm{~s}$
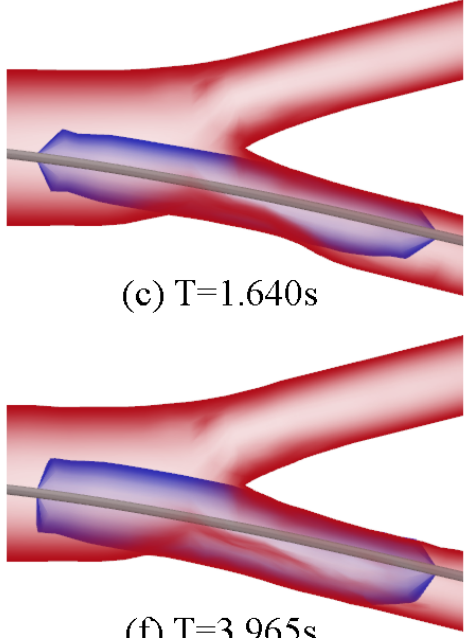

(f) $\mathrm{T}=3.965 \mathrm{~s}$

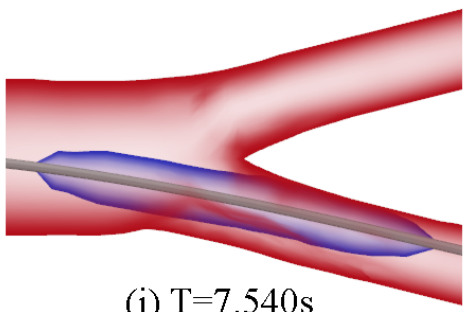

(i) $\mathrm{T}=7.540 \mathrm{~s}$

Figure 10: The regular Y-type stenosed blood vessel dilated by the inflation balloon

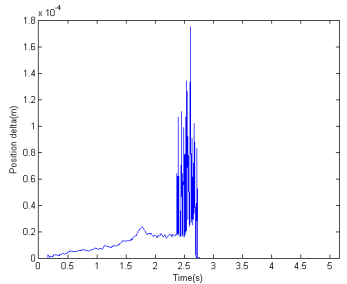

(a) frictional position delta

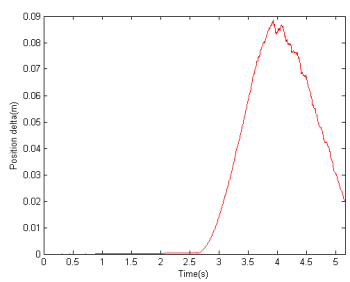

(b) viscous position delta
Figure 12: The frictional and viscous position delta of balloon in real lesion coronary artery

viscous position delta increases firstly and then decreases which is similar with the previous experiments.

Since the entire process of the balloon angioplasty is position based solver, this angioplasty system is parallel solved by CUDA with the frame rate over 40FPS. The experimental environment is listed as following and the details of simulation models in our experiments are shown in Table 2.

- Software environment: CUDA version 8.0;

- Hardware environment: Intel(R) Xeon(R) CPU W3530 @ 2.80GHz, NVIDIA Quadro K5200 and 6GB memory.

\section{Conclusion}

In this paper, we present a novel position-based physical model of balloon for simulating the nonlinear deformation during the process
Table 2: The simulation models

\begin{tabular}{|c|c|c|}
\hline Models & Triangle number & Particle number \\
\hline Quadratic cloth & 5000 & 2601 \\
\hline Unilateral blood vessel & 2400 & 1220 \\
\hline Bilateral blood vessel & 2400 & 1220 \\
\hline Y-type blood vessel & 17600 & 8859 \\
\hline The real coronary artery & 30420 & 15215 \\
\hline The balloon model & 1040 & 522 \\
\hline Guidewire & - & 24 \\
\hline
\end{tabular}

of balloon angioplasty. Considering the material physical characteristics of real balloon, hyperelastic membrane material and continuum based formulation are combined to compute the mechanical properties in the process of balloon inflation. Considering the locality of collision detection between two flexible objects, an adaptive air mesh generation algorithm is put forward to fast generate the collision air meshes for the collision handling. Finally, we designed four different type experiments to validate the balloon model. The experiments results show that the our balloon physical model could simulate the balloon inflation in angioplasty very well in real-time.

In the future, we will continue to improve the balloon physical model and apply it into virtual angioplasty procedures training.

\section{References}

BARAFF, D., WitKin, A., AND Kass, M. 2003. Untangling cloth. In ACM Transactions on Graphics (TOG), vol. 22, ACM, 862-870. 
BARBIČ, J., AND JAMES, D. L. 2010. Subspace self-collision culling. In ACM Transactions on Graphics (TOG), vol. 29, ACM, 81 .

Bender, J., Koschier, D., Charrier, P., And Weber, D. 2014. Position-based simulation of continuous materials. Computers \& Graphics 44, 1-10.

Bouaziz, S., Martin, S., Liu, T., Kavan, L., And Pauly, M. 2014. Projective dynamics: fusing constraint projections for fast simulation. ACM Transactions on Graphics (TOG) 33, 4, 154.

Bridson, R., Fedkiw, R., AND Anderson, J. 2002. Robust treatment of collisions, contact and friction for cloth animation. ACM Transactions on Graphics (TOG) 21, 3, 594-603.

Cai, Y., Chui, C., Ye, X., Wang, Y., And Anderson, J. H. 2003. Vr simulated training for less invasive vascular intervention. Computers \& Graphics 27, 2, 215-221.

Cai, Y., Chui, C.-K., Ye, X., FAn, Z., And Anderson, J. H. 2006. Tactile vr for hand-eye coordination in simulated ptca. Computers in Biology and Medicine 36, 2, 167-180.

CHOI, K.-J., AND Ko, H.-S. 2005. Stable but responsive cloth. In ACM SIGGRAPH 2005 Courses, ACM, 1.

Chua, S. D., Mac Donald, B., And Hashmi, M. 2003. Finite element simulation of stent and balloon interaction. Journal of Materials Processing Technology 143, 591-597.

Cirak, F., Scott, M. J., Antonsson, E. K., Ortiz, M., AND SCHRÖDER, P. 2002. Integrated modeling, finite-element analysis, and engineering design for thin-shell structures using subdivision. Computer-Aided Design 34, 2, 137-148.

Eftaxiopoulos, D. A., And AtKinson, C. 2005. A nonlinear, anisotropic and axisymmetric model for balloon angioplasty. In Proceedings of the Royal Society of London A: Mathematical, Physical and Engineering Sciences, vol. 461, The Royal Society, 1097-1128.

Gasser, T. C., And Holzapfel, G. A. 2007. Finite element modeling of balloon angioplasty by considering overstretch of remnant non-diseased tissues in lesions. Computational Mechanics 40, 1, 47-60.

Grinspun, E., HirAni, A. N., Desbrun, M., AND Schröder, P. 2003. Discrete shells. In Proceedings of the 2003 ACM SIGGRAPH/Eurographics symposium on Computer animation, Eurographics Association, 62-67.

Karimi, A., NavidbaKhsh, M., And Razaghi, R. 2014. A finite element study of balloon expandable stent for plaque and arterial wall vulnerability assessment. Journal of Applied Physics $116,4,044701$.

Luboz, V., Kyaw-Tun, J., Sen, S., Kneebone, R., DickinSON, R., Kitney, R., AND Bello, F. 2014. Real-time stent and balloon simulation for stenosis treatment. The Visual Computer 30, 3, 341-349.

Macklin, M., MÜller, M., Chentanez, N., And Kim, T.-Y. 2014. Unified particle physics for real-time applications. $A C M$ Transactions on Graphics (TOG) 33, 4, 153.

Mezger, J., Kimmerle, S., And Etzmuss, O. 2003. Hierarchical techniques in collision detection for cloth animation.

Müller, M., Keiser, R., Nealen, A., Pauly, M., Gross, M., AND AleXA, M. 2004. Point based animation of elastic, plastic and melting objects. In Proceedings of the 2004 ACM
SIGGRAPH/Eurographics symposium on Computer animation, Eurographics Association, 141-151.

Müller, M., Heidelberger, B., Teschner, M., AND Gross, M. 2005. Meshless deformations based on shape matching. ACM Transactions on Graphics (TOG) 24, 3, 471478.

Müller, M., Heidelberger, B., Hennix, M., And RatclifF, J. 2007. Position based dynamics. Journal of Visual Communication and Image Representation 18, 2, 109-118.

Müller, M., Chentanez, N., Kim, T.-Y., And Macklin, M. 2015. Air meshes for robust collision handling. ACM Transactions on Graphics (TOG) 34, 4, 133.

Naghavi, M., Libby, P., Falk, E., Casscells, S. W., LITOVSKy, S., Rumberger, J., BAdimon, J. J., STEFAnAdis, C., Moreno, P., Pasterkamp, G., et AL. 2003. From vulnerable plaque to vulnerable patient a call for new definitions and risk assessment strategies: part i. Circulation 108, 14, 1664 1672.

Schvartzman, S. C., Gascón, J., and Otaduy, M. A. 2009. Bounded normal trees for reduced deformations of triangulated surfaces. In Proceedings of the 2009 ACM SIGGRAPH/Eurographics Symposium on Computer Animation, ACM, 75-82.

Schvartzman, S. C., Pérez, Á. G., and Otaduy, M. A. 2010. Star-contours for efficient hierarchical self-collision detection. In ACM Transactions on Graphics (TOG), vol. 29, ACM, 80 .

Selle, A., Su, J., Irving, G., And FedKiw, R. 2009. Robust high-resolution cloth using parallelism, history-based collisions, and accurate friction. IEEE Transactions on Visualization and Computer Graphics 15, 2, 339-350.

SHEWCHUK, J. R. 2002. Two discrete optimization algorithms for the topological improvement of tetrahedral meshes. Unpublished manuscript 65.

SI, H. 2015. Tetgen, a delaunay-based quality tetrahedral mesh generator. ACM Transactions on Mathematical Software (TOMS) $41,2,11$.

Skouras, M., Thomaszewski, B., Bickel, B., And Gross, M. 2012. Computational design of rubber balloons. In Computer Graphics Forum, vol. 31, Wiley Online Library, 835-844.

Skouras, M., Thomaszewsi, B., Kaufmann, P., Garg, A., Bickel, B., Grinspun, E., ANd Gross, M. 2014. Designing inflatable structures. ACM Transactions on Graphics (TOG) 33, 4, 63.

Tang, W., Lagadec, P., Gould, D., Wan, T. R., Zhai, J., AND How, T. 2010. A realistic elastic rod model for real-time simulation of minimally invasive vascular interventions. The Visual Computer 26, 9, 1157-1165.

Tang, M., Manocha, D., Yoon, S.-E., Du, P., Heo, J.-P., AND TONG, R.-F. 2011. Volccd: Fast continuous collision culling between deforming volume meshes. ACM Transactions on Graphics (TOG) 30, 5, 111 .

TANG, M., Tong, R., WAng, Z., And Manocha, D. 2014. Fast and exact continuous collision detection with bernstein sign classification. ACM Transactions on Graphics (TOG) 33, 6, 186.

TANG, W., WAN, T. R., AND HUANG, D. 2015. Interactive thin elastic materials. Computer Animation and Virtual Worlds. 
Terzopoulos, D., Platt, J., Barr, A., And Fleischer, K. 1987. Elastically deformable models. In ACM Siggraph Computer Graphics, vol. 21, ACM, 205-214.

Teschner, M., Heidelberger, B., Müller, M., PomerANTES, D., AND GRoss, M. H. 2003. Optimized spatial hashing for collision detection of deformable objects. In $V M V$, vol. 3 , $47-54$.

Volino, P., and Magnenat-Thalmann, N. 2006. Resolving surface collisions through intersection contour minimization, vol. 25. ACM.

Volino, P., Magnenat-Thalmann, N., And Faure, F. 2009. A simple approach to nonlinear tensile stiffness for accurate cloth simulation. ACM Transactions on Graphics 28, 4, ArticleNo.

Wang, Q., LiU, X., LU, X., CAO, J., AND TANG, W. 2015. Cute balloons with thickness. In International Conference on Image and Graphics, Springer, 75-89.

WANG, H. 2014. Defending continuous collision detection against errors. ACM Transactions on Graphics (TOG) 33, 4, 122.

Wicke, M., LANKer, H., AND Gross, M. 2006. Untangling cloth with boundaries. In Proc. of Vision, Modeling, and Visualization, 349-356.

Wu, X., Downes, M. S., Goktekin, T., And Tendick, F. 2001. Adaptive nonlinear finite elements for deformable body simulation using dynamic progressive meshes. In Computer Graphics Forum, vol. 20, Wiley Online Library, 349-358.

Zahedmanesh, H., Kelly, D. J., ANd Lally, C. 2010. Simulation of a balloon expandable stent in a realistic coronary arteryłdetermination of the optimum modelling strategy. Journal of Biomechanics 43, 11, 2126-2132.

ZHANG, X., AND KIM, Y. J. 2012. Simple culling methods for continuous collision detection of deforming triangles. IEEE transactions on visualization and computer graphics $18,7,1146$.

ZHENG, C., AND JAMES, D. L. 2012. Energy-based self-collision culling for arbitrary mesh deformations. ACM Transactions on Graphics (TOG) 31, 4, 98 .

\section{A Derivation of The first Piola-Kirchhoff stress tensor.}

In Equation(17), the every parts of first Piola-Kirchhoff stress tensor $\mathbf{P}(\mathbf{F})$ are:

$$
\begin{gathered}
\frac{\partial \Psi}{\partial I_{1}}=\frac{\partial \Psi}{\partial \lambda_{1}} \frac{\partial \lambda_{1}}{\partial I_{1}}+\frac{\partial \Psi}{\partial \lambda_{2}} \frac{\partial \lambda_{2}}{\partial I_{1}}+\frac{\partial \Psi}{\partial \lambda_{3}} \frac{\partial \lambda_{3}}{\partial I_{1}} \\
\frac{\partial \Psi}{\partial I_{3}}=\frac{\partial \Psi}{\partial \lambda_{1}} \frac{\partial \lambda_{1}}{\partial I_{3}}+\frac{\partial \Psi}{\partial \lambda_{2}} \frac{\partial \lambda_{2}}{\partial I_{3}}+\frac{\partial \Psi}{\partial \lambda_{3}} \frac{\partial \lambda_{3}}{\partial I_{3}} \\
\frac{\partial I_{1}}{\partial \mathbf{F}}=2 \mathbf{F} \\
\frac{\partial I_{3}}{\partial \mathbf{F}}=2\left[\begin{array}{ll}
A_{11} & A_{12} \\
A_{21} & A_{22} \\
A_{31} & A_{32}
\end{array}\right] \\
A_{11}=\mathbf{F}_{11} \mathbf{F}_{22}^{2}+\mathbf{F}_{11} \mathbf{F}_{32}^{2}-\mathbf{F}_{12} \mathbf{F}_{21} \mathbf{F}_{22}-\mathbf{F}_{12} \mathbf{F}_{31} \mathbf{F}_{32}
\end{gathered}
$$

$$
A_{12}=-\mathbf{F}_{11} \mathbf{F}_{21} \mathbf{F}_{22}-\mathbf{F}_{11} \mathbf{F}_{31} \mathbf{F}_{32}+\mathbf{F}_{12} \mathbf{F}_{21}^{2}+\mathbf{F}_{12} \mathbf{F}_{31}^{2}
$$

$A_{21}=-\mathbf{F}_{11} \mathbf{F}_{12} \mathbf{F}_{22}+\mathbf{F}_{12}^{2} \mathbf{F}_{21}+\mathbf{F}_{21} \mathbf{F}_{32}^{2}-\mathbf{F}_{22} \mathbf{F}_{31} \mathbf{F}_{32}$

$$
A_{22}=\mathbf{F}_{11} \mathbf{F}_{22}^{2}-\mathbf{F}_{11} \mathbf{F}_{12} \mathbf{F}_{21}-\mathbf{F}_{21} \mathbf{F}_{31} \mathbf{F}_{32}+\mathbf{F}_{22} \mathbf{F}_{31}^{2}
$$

$$
A_{31}=-\mathbf{F}_{11} \mathbf{F}_{12} \mathbf{F}_{32}+\mathbf{F}_{12}^{2} \mathbf{F}_{31}-\mathbf{F}_{21} \mathbf{F}_{22} \mathbf{F}_{32}-\mathbf{F}_{22}^{2} \mathbf{F}_{31}
$$

$$
A_{32}=\mathbf{F}_{11}^{2} \mathbf{F}_{32}-\mathbf{F}_{11} \mathbf{F}_{12} \mathbf{F}_{31}+\mathbf{F}_{21}^{2} \mathbf{F}_{32}-\mathbf{F}_{21} \mathbf{F}_{22} \mathbf{F}_{31}
$$

For $I_{1}$ we have:

$$
\begin{gathered}
\frac{\partial \lambda_{1}}{\partial I_{1}}=\frac{1}{2}\left(I_{1}+\left(I_{1}^{2}-4 I_{3}\right)^{1 / 2}\right)^{-1 / 2} \cdot\left(1+\left(I_{1}^{2}-4 I_{3}\right)^{-1 / 2} I_{1}\right) \\
\frac{\partial \lambda_{2}}{\partial I_{1}}=\frac{1}{2}\left(I_{1}-\left(I_{1}^{2}-4 I_{3}\right)^{1 / 2}\right)^{-1 / 2} \cdot\left(1-\left(I_{1}^{2}-4 I_{3}\right)^{-1 / 2} I_{1}\right) \\
\frac{\partial \lambda_{3}}{\partial I_{1}}=-\lambda_{1}^{-2} \lambda_{2}^{-1} \frac{\partial \lambda_{1}}{\partial I_{1}}-\lambda_{2}^{-2} \lambda_{1}^{-1} \frac{\partial \lambda_{2}}{\partial I_{1}}
\end{gathered}
$$

For $I_{3}$ we have:

$$
\begin{gathered}
\frac{\partial \lambda_{1}}{\partial I_{3}}=-\left(I_{1}+\left(I_{1}^{2}-4 I_{3}\right)^{1 / 2}\right)^{-1 / 2} \cdot\left(I_{1}^{2}-4 I_{3}\right)^{-1 / 2} \\
\frac{\partial \lambda_{2}}{\partial I_{3}}=\left(I_{1}-\left(I_{1}^{2}-4 I_{3}\right)^{1 / 2}\right)^{-1 / 2} \cdot\left(I_{1}^{2}-4 I_{3}\right)^{-1 / 2} \\
\frac{\partial \lambda_{3}}{\partial I_{3}}=-\lambda_{1}^{-2} \lambda_{2}^{-1} \frac{\partial \lambda_{1}}{\partial I_{3}}-\lambda_{2}^{-2} \lambda_{1}^{-1} \frac{\partial \lambda_{2}}{\partial I_{3}}
\end{gathered}
$$

